\title{
Le parasitisme du zébu dans l'ouest de la République Centrafricaine I. - Parasitisme des veaux de lait
} por A. BOUCHET, M. GRABER, P. FINELLE, J. DESROTOUR ef G. MACON (avec la collaboration technique de Madame MACON)

\begin{abstract}
RÉSUMÉ
Dans l'Ouest de la République Centrafrıcaine, les auteurs signalent l'existence, chez les veaux de lait de 0 à 7 mois, de nombreux parasites, seuls ou associés entre eux, appartenant aux espèces sulvantes : Neoascaris vitulorum, Strongyloides papillosus, Oesaphagostomum (Bosicala) radiatum, Bunostomum phlebotomum, Cooperia punctato et Cooperia pectinata, Haemoncus contortus et diverses Coccidies.

Les animaux sont atteints dans la proportion de 76,4 p. 100. Les taux d'infestation et la nature du parasıtisme varient en fonction de l'âge ef de la saison. La mortalité oscille entre 1 et 20 p. 100 , selon les troupeaux et les années.

II s'agit là, pour l'Elevage de la République Centrafricaine, d'un problème majeur, à résoudre d'urgence.
\end{abstract}

\section{GÉNÉRALITÉS}

\section{1. - Le pays.}

La région intéressée est comprise entre les $4 \mathrm{e}$ ef 8 e degrés de latitude Nord et les 15 et 17 e degrés de longitude Est.

Elle est formée au Nord par un massif montagneux, le Yadé, dont l'altitude moyenne varie entre 900 et 1.200 mètres (point culmınant le Gaou 1.420 mètres) et qui se prolonge vers l'Est et le Sud par des plateaux compris entre 500 ef 800 mètres.

Le réseau hydrogrophique, très dense, alımente deux bassins :

- celui du Congo avec la Kades, la Mambéré et la Nana qui, réunies, constituent la Sangha. Plus à l'Est, la Lobaye reçoit la Topıa et se jette dans l'Oubangui à la frontière congolaise ;

- celui du Tchad avec la Lim (l'une des branches du Logone occidental), la Pende(Logone oriental), l'Ouham et la Nana Barya qui donnent au-delà de la frontière tchadienne le BahrSara.

La partie Nord de cette région représente donc le versant sud-ouest du bassın tchadien et, à ce tıtre, Il est ıntéressant d'établır une comparaison avec ce qui est observé dans la moyenne vallée du Logone (Moundou), de manière à préciser pour l'ensemble du bassin du Logone, la nature exacte du parasitisme des bêtes à cornes.

L'enquête a couvert les préfectures de Bovar, de l'Ouham-Pendé et du Nord de la HauteSangha.

\section{2. - Climat et végétation.}

D'une façon générale, le climat esł de type intertropical (CHAARA, 1962; GOULÉE, 1964) avec une sasson des pluies et une saison sèche bien marquées (la saison des pluies est plus longue que la sason sèche), une moyenne 


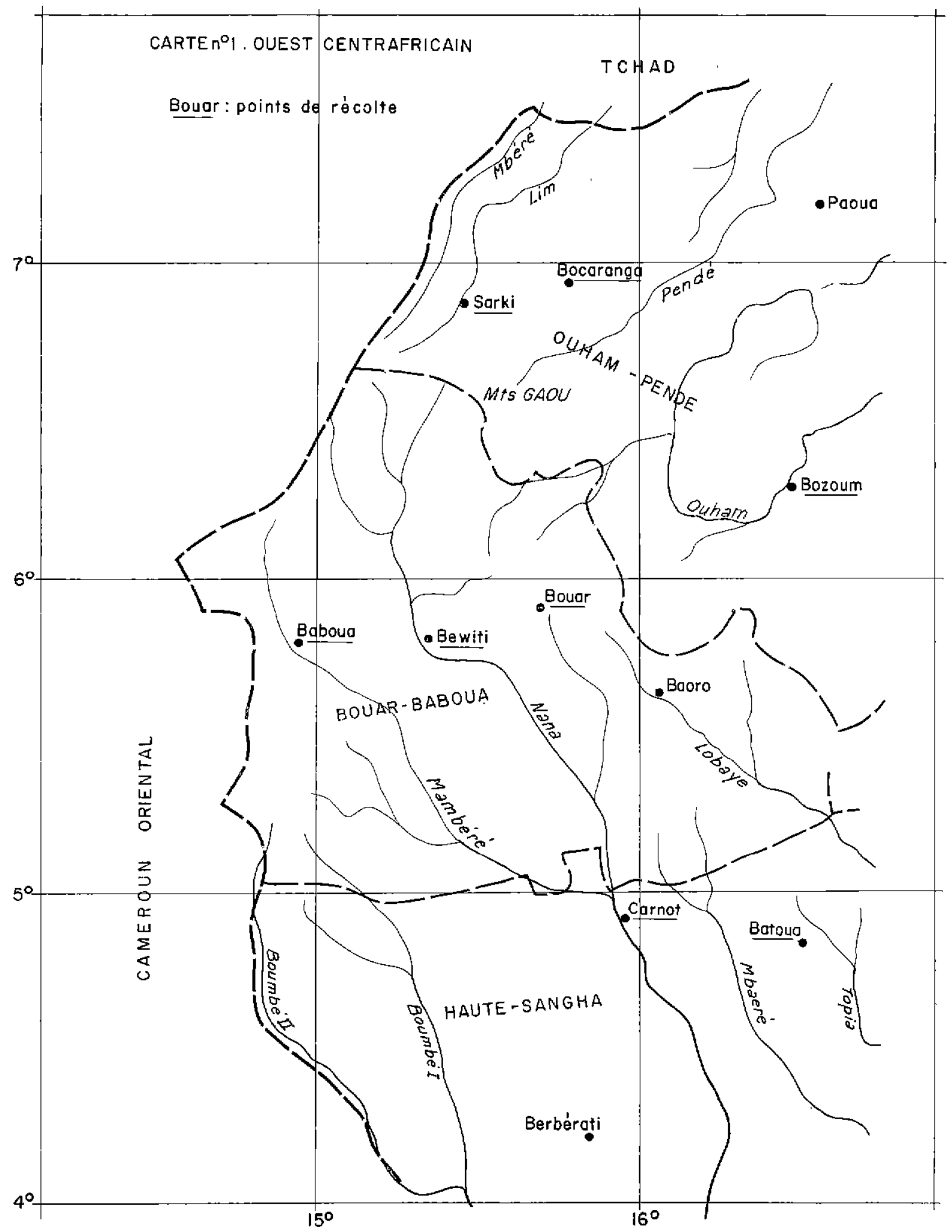


annuelle des précipitatıons comprise entre 1.350 et $1.600 \mathrm{~mm}$, une amplitude des variations thermiques et hygrométriques relativement forte en saison sèche, plus faible en saison des pluies.

Les différences s'accentuent au fur et à mesure que I'on remonte vers le Nord:

- entre le $4^{\mathrm{e}}$ et le $6^{\mathrm{e}}$ parallèle, c'est la zone guinéenne préforestıère (Carnot-Berbératı). La pluviométrie dépasse $1.500 \mathrm{~mm}$. La saison sèche commence en décembre et ne dure que 3 mois. L'humidité moyenne oscille entre $48-55$ p. 100 et $86-97$ p. 100 , et la température entre 19 et $30^{\circ} \mathrm{C}$. Le nombre de jours de pluie est voisin de 140.

C'est une région de savanes bolsées, coupées d'importantes galeries forestières, assez favorables à l'élevage.

- Au Nord du 6e parallèle, le climat et la végétation sont de type soudano-guinéen (Sarki - frontıère Tchad - RCA). Les précipitations annuelles sont inférieures à $1.400 \mathrm{~mm}$ avec 90 jours de pluie. La saison sèche devient de plus en plus longue ( 5 mois envıron à Bocaranga, GOULÉE, 1964).

Les conditions climatiques sont donc éminemment favorables à l'évolution et à la pulluJation des parasites.

\section{3. - Le bétail.}

L'élevage, dans l'Ouest centrafricain, est de création récente. Ce n'est qu'en 1924 que les premiers pasteurs Bororos, venus du Cameroun, s'installent dans les savanes du massif du Yadé. Sur ces pâturages neufs, les troupeaux se multiplient rapidement et en 1933, le cheptel était estimé à 150.000 têtes.

L'important accroissement du troupeau incite alors les éleveurs à rechercher de nouveaux pâturages : ils gagnent les zones plus basses, infestées de glossines et la trypanosomiase commence d̀ sévir, ce qui amène en 1939 un regroupement du cheptel au Nord-Ouest de Bouar, à proxımıté de la frontıère camerounaıse, là où l'altıtude moyenne dépasse 1.000 mètres.

A partir de 1954, le service de l'Elevage intervient vigoureusement et l'intensification de la lutte contre la trypanosomiase, par traitement des animaux atteints ou par stérilisation de cer- taines zones à glossines (Topia) entraîne une importante progression du bétaıl.

Malheureusement, en 1958, I'apparition de la péripneumonie faillit tout compromettre et impose encore à l'heure actuelle des mesures sanitaires sévères (regroupement des troupeaux infectés: vaccination, etc.).

Malgré ces aléas, les zébus sont actuellement dans l'Ouest centrafricain au nombre de 400.000, répartis ainsi :

\section{Bouar : 380.000 .}

Topia (Djomo) : 13.000.

Yaloké : 7.000 .

Désirant assurer une augmentation continue du cheptel, le Service de l'Elevage de RCA devait se préoccuper, dès 1959, du problème des helminthiases gastro-intestinales des jeunes let des adultes qui, en Afrique centrale, constituent un obstacle sérieux au développement de l'élevage. Une première mission en 1959 (GRABER, 1961) permettait de faire le point de la situation. Une enquête plus approfondie était décidée ef le Service de l'Elevage affectait en 1962 un agent à l'abattoir de Bouar dont la mission était de procéder à la récolte des helmınthes prélevés sur les animaux sacrifiés pour la boucherie.

Plus récemment, le Gouvernement centrafricain demandait au laboratoire de Farcha d'étudier une maladie grave existant dans la région de la Topia, et connue sous le nom de «Bebou».

Les résultats de l'enquête effectuée sur place indiquaient la présence, surtout chez les jeunes, d'un grand nombre de parasites internes, souvent dangereux, et dont l'action se traduit par une morbidıté importante et une mortalıté quelquefois élevée (GRABER, 1968 b).

Aussi, devant la gravité de la situation, une section d'Helminthologie étalt-elle créée à Bouar avec mission, en priorité, de poursuivre l'enquête sur le parasitisme des veaux de lait.

Le but du présent travall est de tenter de regrouper les observations faites depuis une dizaine d'années dans l'Ouest de la RCA.

\section{MATÉRIEL ET MÉTHODE}

\section{1. - Veaux de lait.}

Ont été examinés 1.165 veaux de 1 jour à 7 mois, originaires des localités suivantes: Baboua, Sarki, De Gaulle, Bouar, Carnot, 
Batoua (carte no 1). Quelques autopsies ont été faites à Bouar, pour préciser les espèces parasites les plus fréquemment rencontrées.

La méthode utilısée est celle des examens coproscopiques par sédimentation ou por flottaison (solution d'iodo-mercurate de potassium et méthode de MAC MASTER'modifiée par GORDON ef WHITLOCK en solution de sulfate de zınc à 33 p. 100).

\section{2. - Bouvillons.}

846 examens coproscopiques ont été effectués chez des animaux de 7 mois à 3 ans (Baboua, Bozoum, Bouar, Batoua et Bocaranga).

En outre, de 1964 à 1969, 150 bouvillons, achetés à Béwiti, Sarki, Baboua et Bocaranga ont été autopsiés au laboratoire de Farcha où ils avaient été transportés en vue de certaines expériences, sur la peste bovine notamment.

\section{3. - Zébus adultes.}

L'enquête a porté sur 662 animaux sacrifiés aux abattoirs de Bouar et de Carnot de 1962 à 1967. Les parasites ont été recueillıs sur place selon les techniques habituelles (EUZÉBY,1958) et expédiés au laboratoire où Ils ont été déterminés, pesés ou comptés.

\section{RÉSULTATS}

En matière d'helminthiases du zébu, :on :distingue, en Afrique centrale, trois étages de parasitisme :

- celui des veaux de lait,

- celui des bouvillons ( 7 mols à 2 ans et demi),

- celui des adultes.

On retrouve cette distinction dans l'Ouest centrafricain où la nature et l'importance des verminoses gastro-intestinales varient en fonction de l'âge et du milieu.

\section{I. - Parasitisme des veaux de lait.}

Les animaux ont été divisés en plusieurs catégories :

- vecux de 0 à 15 jours,

- vecux de 15 jours à 1 mois,

— veaux de 1 mois à 2 mois,

- veaux de 2 mois à 3 mois,

- veaux de 3 mois à 7 mois.

\section{2. - Nature de l'infestation parasitaire.}

\subsection{1. - Infestation globale.}

Un certain nombre de parasites, surtout des nématodes, ont été mis en évidence dans le tractus digestıf : ce sont :

Neooscaris vifulorum (GOEZE, 1782) :

Strongyloides papillosus (WEDL, 1856) ;

Oesophagostomum (*) (Bosicola radiatum) (RUDOLPHI, 1803):

Bunostomum phlebotomum (RAILLIET, 1900) ;

Cooperio punctata (VON LINSTOW, 1907);

Cooperia pectinato (RANSOM, 1907):

Haemoncus contortus (RUDOLPHI, 1803) ;

Diverses coccidies.

Le téniasis par Moniezia et Thysaniezia est négligeable (moins de 2 p. 100 sur la Topia).

61,9 p. 100 des veaux sont infestés dans les quinze premiers jours de leur existence. Le pourcentage d'animaux atteints passe par un maximum à l'âge de 2 mois $(84,3$ p. 100), puis redescend' progressivement de 3 à 6 mois jusqu'à 70 p. 100 (tableau no 1).

\section{TABLEAU $\mathbb{N}^{\circ}$ I}

Taux moyen d'infestation des veaux de l'ouest de la Sépublique Centrafrícaine Années 1963 à 1969

\begin{tabular}{|c|c|c|c|}
\hline $\begin{array}{c}\text { Age des } \\
\text { veaux }\end{array}$ & $\begin{array}{c}\text { Nombre } \\
\text { d'animaux } \\
\text { examinés }\end{array}$ & $\begin{array}{c}\text { Nombre } \\
\text { d'enimaux } \\
\text { parasités }\end{array}$ & $\begin{array}{c}\text { Pourcentage } \\
\text { d'infestation }\end{array}$ \\
\hline $0-15$ jours & 92 & 57 & 61,9 p.100 \\
\hline 5 jours-1 mois & 190 & 157 & 82,6 \\
\hline $1-2$ mois & 320 & 270 & 84,3 \\
\hline $2-3$ mois & 212 & 165 & 77,8 \\
\hline $3-7$ mois & 351 & 242 & 68,9 \\
\hline Total & 1165 & 891 & 76,4 \\
\hline
\end{tabular}

En médecine vétérinaire, il est classique de considérer qu'un taux d'infestation parasitare ne signifie pas grand-chose, car les animaux hébergent fréquemment des parasites qui ne paraissent pas les gêner outre mesure.

(*) Pour la commodité de l'exposé, Oesophagostomum, Bunostomum, Cooperia ef Hoemoncus seront désignés sous le nom de « Strongles». 


\section{Graphique $n^{\circ} \mid$ : TAUX DíNFESTATION DES VEAUX DE}

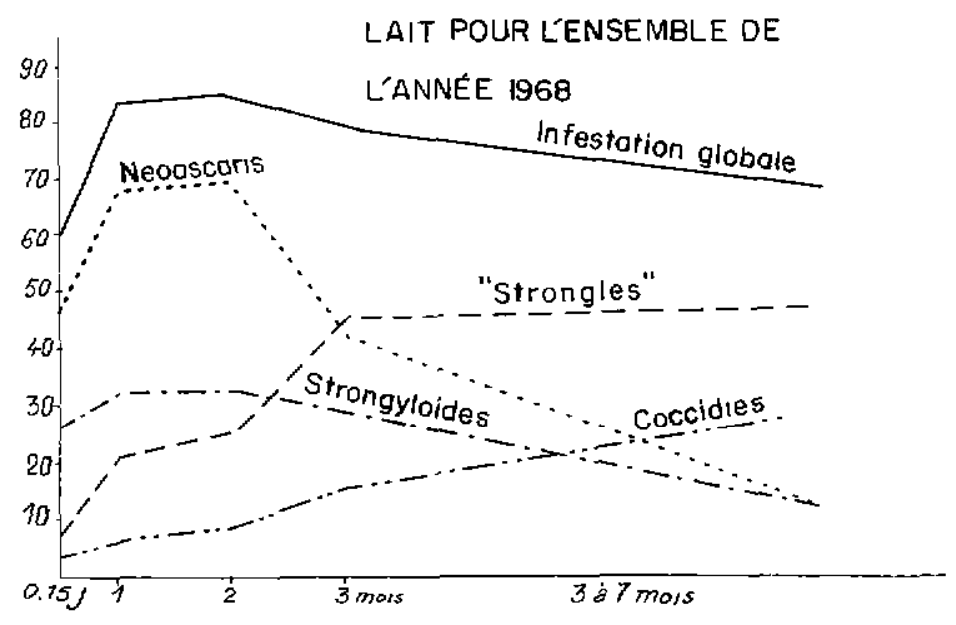

En milieu tropical, il n'en est pos de même, et le taux d'infestotion semble correspondre à peu près au pourcentage de veaux dont l'état clinique et la croissance nécessitent un traitement anthelminthique.

\subsection{2. - Infestation par Neoascaris vitulorum.}

Le nombre de jeunes porteurs d'ascaris progresse régulièrement depuis la naissance jusqu'à l'âge de 2 mois (tableau $n^{\circ} 2$ ). Les nématodes sont ensuite expulsés naturellement et le pourcentage tombe à environ 13,6 p. 100 au-delà de 3 mois : le nombre de parasites rencontrés à l'autopsie est alors très faible (quelques unités). Par contre, entre 3 semaines et 2 mois, l'infestation est en général massive.

47,8 p. 100 des veaux de 0 à 15 jours hébergent des ascaris, ce qui est imputable à l'infestation prénatale, la migration de Neooscaris vitulorum étant de fype entéro-pneumo-somatique (CVE?KOVIC et NEVENIC, 1960). L'infestation postnatale, rarissime, ne peut se produire que les premières heures de la vie quand le jeune n'a pas encore absorbé le colostrum maternel. Ce fait mériterait d'être confirmé en milieu tropical humide.

Ce taux, basé sur les examens coproscopiques positifs est sans doute loin de correspondre à la réalité car les ascaris n'ont pas tous atteint le stade de maturité sexuelle $\left({ }^{*}\right)$, et les œufs ne peuvent donc être décelés au microscope.

(*) 3 semaines environ après la naissance du veau.
Cependant, la présence d'œufs de Néoascaris dans les fèces d'un certain nombre de veaux de moins d'une semaine laisse supposer que quelquefois, la dernière migration des larves d'ascaris par le poumon, la trachée, l'estomac et l'intestin du foetus est susceptible, en milieu tropical contaminé, de se produire avant la naissance et non après comme il est classiquement admis.

1.1.3. - Infestation par Strongyloides papillosus.

La strongyloidose est également une affection sérieuse dont il ne faut pas sous-estimer l'importance.

C'est essentiellement une maladie des veaux de moins de 3 mois. Passé cet âge, l'ocquisition d'une résistance spécifique se traduit par une diminution sensible du taux de parasites.

Un essaı réalisé sur une «corde de veaux de 25 anımaux environ », débarrassés de leurs ascaris, mais non de leurs angullules, a permis en outre de préciser quelques points concernant la symptomatologie de la strongyloïdose bovine.

Elle s'accompagne d'une diarrhée profuse, jaunâtre, lientérique, parfois mucoıde et d'odeur fétide. L'amaigrissement est intense. Le veau est souffreteux, faible et suit péniblement le troupeau. On note une forte anémie hypochrome.

Ces signes peuvent prêter à confusion avec une septicémie en phase débutante. 


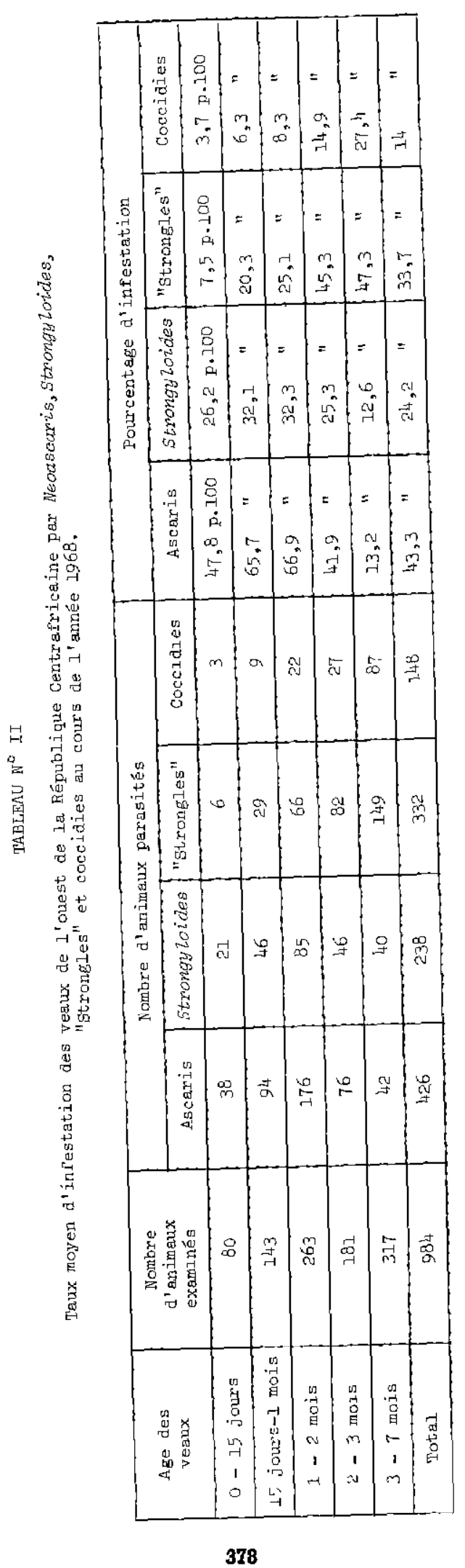


1.1.4. - Infestation por les «Strongles».

Le pourcentage d'infestation augmente régulièrement depuls la naissance pour atteindre 47 p. 100 au-delà de 3 mois.

A cet age, les «Strongles» prennent nettement le relais des ascaris.

La moyenne du nombre d'œufs au gramme de matière fécale, chez un sujet parasite, est fonction de l'âge, je la réceptivité de l'animal et de la saıson (la période prépatente de l'ınfestation est généralement plus longue pour les «Strongles » que pour les Strongyloides).

\subsection{5. - Infestation par les Coccidies.}

La coccidiose des veaux de lait de l'Ouest centrafricain est loin d'être négligeable. C'est une maladie saisonnıère, ırrégulièrement répartie, pouvant parfois prendre une allure brutale. Les pertes sont alors élevées.

\subsection{6. - Associations parositoires.}

Les nématodes et les coccidies dont il vient d'être question, sont souvent associés entre eux et il est difficile, sauf pour la coccidiose, de définir une parasitose à l'état pur.

C'est ainsi qu'à Batoua en 1967 (GRABER, 1968) sur 96 veaux examınés, ces parasites se trouvaient associés entre eux dans 50 p. 100 des cas, selon les modalités suivantes :

- Ascaris et Strongyloides : 13.

- Ascaris ef «Strongles》: 7.

— «Strongles » et Strongyloides : 14.

— Ascaris, «Strongles » et Strongylordes : 7.

- Ascaris et Coccidies : 1.

— «Strongles », Strongyloides ef Coccidies : 1.

- «Strongles» et Coccidies : 1 .

- Ascaris, Strongyloides et Coccidies : 2.

— «Strongles» et Cestodes : 2 .

\section{2. - Dynamique de I'infestation. Influence du milieu.}

\subsection{1. - Infestation globale.}

Le nombre d'animaux porteurs de parasites est plus élevé en saison des pluies qu'en saıson sèche (tableau no 3 et graphique no 2). Cette différence est liée à une plus grande prolifération des «Strongles» et des Strongyloides en saison des pluies.

En saison sèche, l'infestation maximale s'ob- serve entre un et deux mois ; elle diminue ensuite.

En sasson des pluies, c'est le contrare qui se produit : le parasitisme gastro-intestinal augmente régulièrement depuis la nassance.

\subsection{2. - Infestation par Neoascaris.}

Le taux d'infestation semble peu influencé par la saison : 41,9 p. 100 en saison des pluies ef 43,3 p. 100 en saison sèche (tableau 4 et graphique 3).

\subsection{3. - Infestation par Strongyloides.}

La saison des pluies est très favorable à l'évolution et à la pullulation de ce nématode : 42,3 p. 100 d'animaux atteints avec une moyenne de 2.090 cufs par gramme (tableau no 6) en saison des pluies, contre 17,1 p. 100 et 517 œufs en saison sèche (tableau no 4 et graphique nं 3).

\subsection{4. - Infestation por les «strongles》.}

Elle est de 54,7 p. 100 en saison des pluies, ef de 25,6 p. 100 en saison sèche, où, à partir de 3 mois, la «Strongylose » semble se stabiliser.

En saison des pluies, les «Strongles», bénéficiant de possibilités d'évolution excellentes (degré hygrométrique élevé, température modérée, chutes de pluies régulièrement espacées et nombreuses), se multiplient à tel point que, vers 3 à 7 mois, 90 p. 100 des jeunes hébergent Haemoncus, Bunostomes, Esophagostomes ou Cooperia, seuls ou associés. (Graphique no 4 . tableau $n^{\circ}$ 5.)

La moyenne du nombre d'ceufs est alors de 607 au gramme, contre 238 en saison sèche (tableau no 6).

\subsection{5. - Infestation por les Coccidies.}

La coccidiose clinique se voit en saison des pluies, plus rarement en saison sèche. Elle se manifeste surtout à partir de 3 mois (tableau no 5, graphique no 4).

\section{3. - Incidence sur l'élevage.}

Les parasites du veau de lat exercent sur l'organisme de leur hôte diverses actions bien connues : mécanique (occlusions intestinales dues à des pelotes d'ascoris), traumatique (Fsophagostomes et Strongylordes), spoliatrice (Haemoncus ef Bunostomes).

Le métabotısme des divers principes alimen- 
TABLEAU $N^{\circ}$ III

Taux d'infestation globale en saison sèche et en saison des pluies 1968.

\begin{tabular}{|c|c|c|c|c|c|c|}
\hline \multirow{2}{*}{$\begin{array}{l}\text { Age des } \\
\text { verux }\end{array}$} & \multicolumn{2}{|c|}{ Nombre de veaux examinés } & \multicolumn{2}{|c|}{ Norbre de veaux parasités } & \multicolumn{2}{|c|}{ Pourcentage d'infestation } \\
\hline & Sazson sècre & $\begin{array}{c}\text { Saison } \\
\text { des pluies }\end{array}$ & Saison sèche & $\begin{array}{c}\text { Salsor } \\
\text { des pluies }\end{array}$ & Saison sèche & $\begin{array}{c}\text { Seison } \\
\text { des pluies }\end{array}$ \\
\hline $0-15$ jours & 48 & 32 & 29 & 21 & 60,4 p. 100 & 65,6 p. .100 \\
\hline 15 joúrs-1 mois & 103 & 40 & 21 & 35 & 78,6 & 87,5 \\
\hline 1 - 2 mois & 186 & 75 & 164 & 69 & 87,2 & 92 \\
\hline $2-3$ молз & 134 & 47 & 97 & $4 \hat{c}$ & 72,3 & 89,4 \\
\hline $3-7$ mois & 237 & 80 & 134 & 79 & 56,5 & 98,8 \\
\hline Tota? & 710 & 274 & 505 & 246 & 72,5 & 89,8 \\
\hline
\end{tabular}

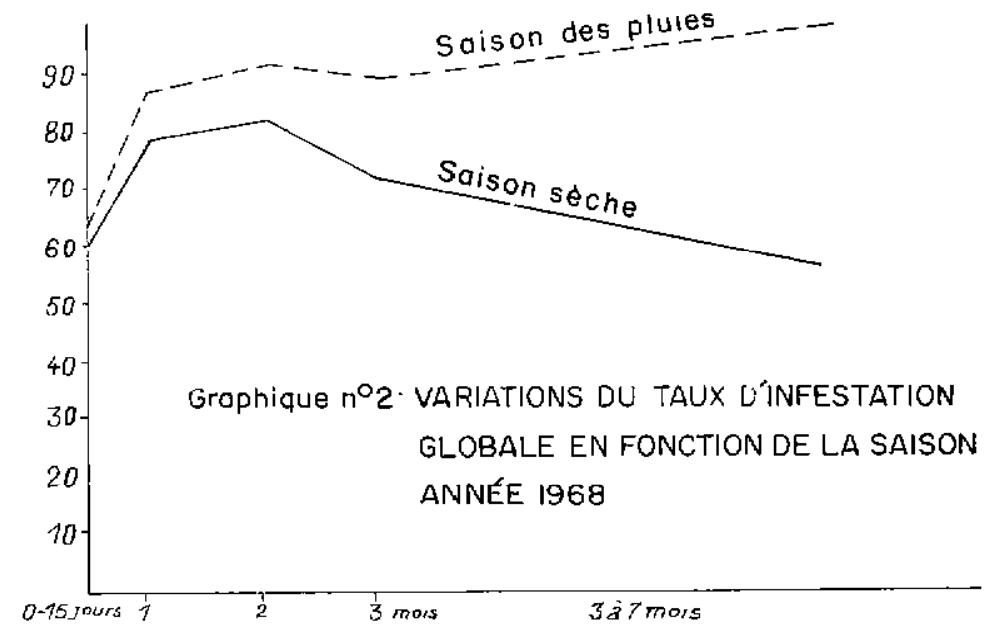

TABLESU $N^{\circ}$ IV

Taux d'infestation par heoascaris et par Strongyzoides en salson sèche et en saison des pluies 1968.

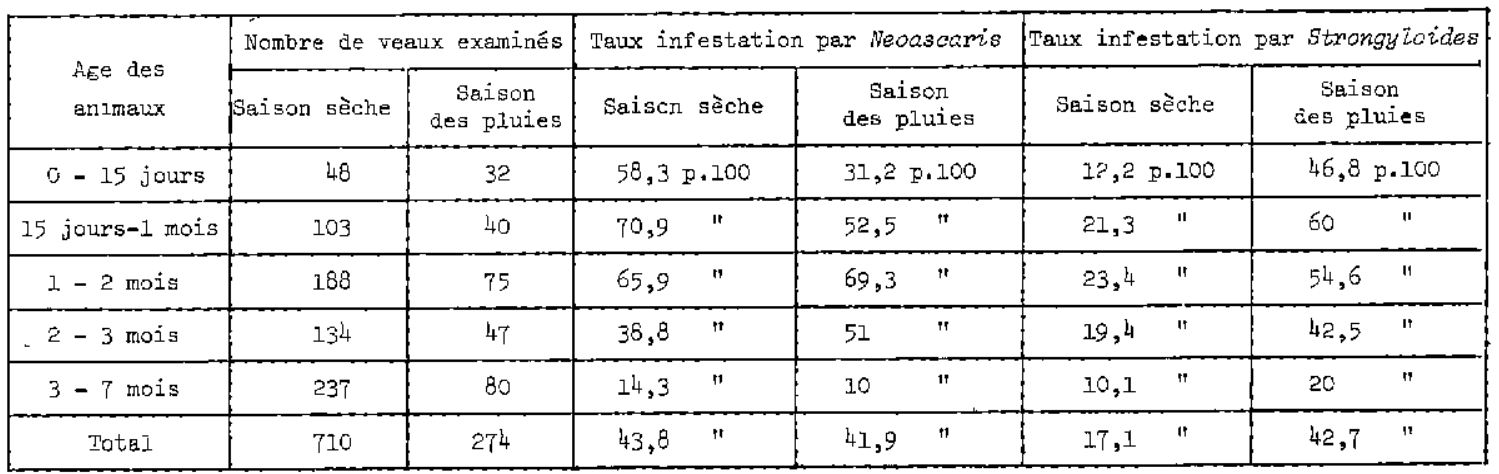




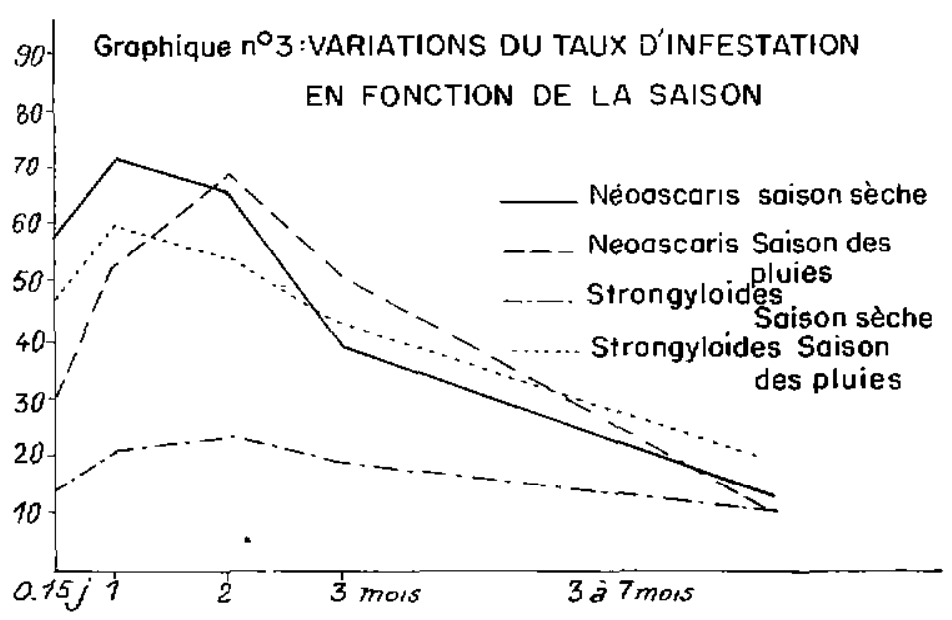

TABLFAU $\mathrm{N}^{\circ} \mathrm{V}$

Teux a'infestation par "Strongles" et coccidies en sauson sèche et en saison des pluzes 1968.

\begin{tabular}{|c|c|c|c|c|c|c|}
\hline \multirow{2}{*}{$\begin{array}{l}\text { Age des } \\
\text { animeux }\end{array}$} & \multicolumn{2}{|c|}{ Nombre de veaux examinés } & \multicolumn{2}{|c|}{ Taux infestations par "Strongles" } & \multicolumn{2}{|c|}{ Taux infestation per Cocciaies } \\
\hline & Saison sèche & $\begin{array}{c}\text { Saison } \\
\text { des pluies }\end{array}$ & Saison sèche & $\begin{array}{c}\text { Sauson } \\
\text { des pluies }\end{array}$ & Srison sèche & $\begin{array}{c}\text { Saison } \\
\text { des pluies }\end{array}$ \\
\hline 0 - 15 jours & 48 & 32 & $6,2 p \cdot 100$ & $9,3 p \cdot 100$ & $\mathrm{p} \cdot 100$ & $9,4 \mathrm{p} \cdot 100$ \\
\hline 15 jours-I mois & 103 & 40 & 13,5 & $37,5 \quad 1$ & 0 & 22,5 \\
\hline $1-2$ mois & 188 & 75 & 19,7 & $38,6 \quad "$ & 6,9 & 12 \\
\hline $2-3$ mois & 134 & 47 & 38 & 65,9 & 13,4 & 19,1 \\
\hline $3-7$ mois & 237 & 80 & 32,4 & 90 & 18,9 & 40 \\
\hline Totel & 710 & 274 & 25,6 & 54,7 & 10,7 & 22,6 \\
\hline
\end{tabular}

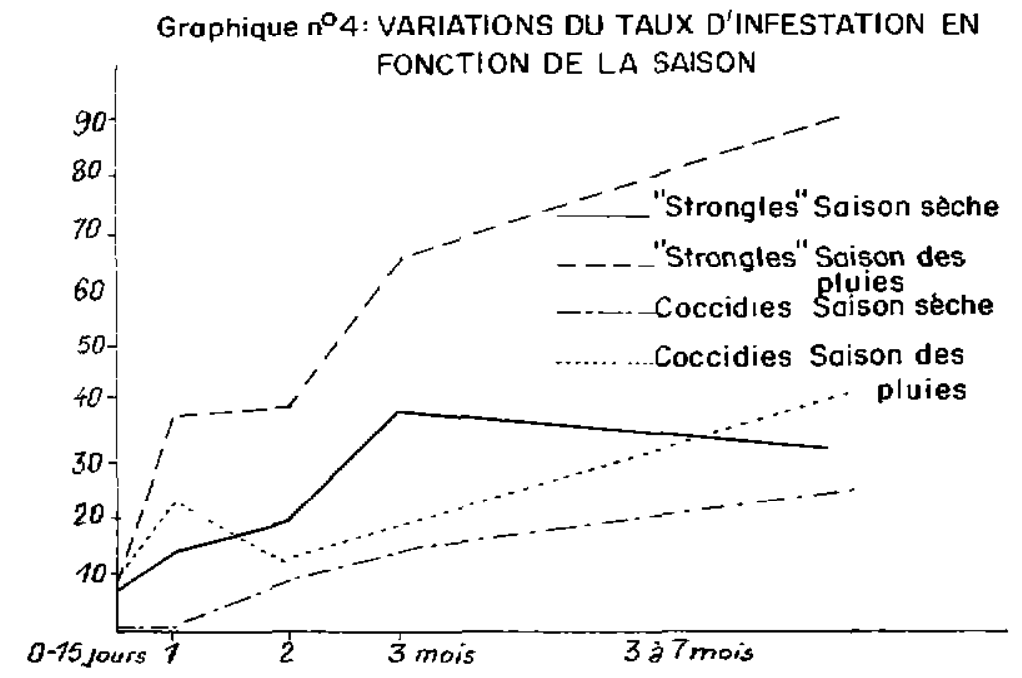


TABLEAU $\mathbb{N}^{\circ}$ VI

Moyenne du nombre d'oeufs au gramme de fèces.

\begin{tabular}{|c|c|c|}
\hline $\begin{array}{c}\text { Equque } \\
\text { Année } 1968\end{array}$ & Strongy Zoides & "Strongles" \\
\hline Saison sèche & 517 & 238 \\
\hline Saison des pluies & 2090 & 607 \\
\hline Ensemble de l'année & 1284 & 401 \\
\hline
\end{tabular}

taires est gravement perturbé. On note une diminution de la digestibilité des glucides et des protides alimentaires aboutissant à de l'hypoglycémie et à un déséquilibre de la protéinémie, avec inversion du rapport albumine/globulines.

La spoliation en fer est très importante dans certains cas (Bunostomes-Haemoncus). Du fait de la diarrhée, phosphore, calcium, potassium et magnésium sont éliminés en quantıté excessive. Certaines vitamines non transformées (vitamine A) ou trop largement prélevées (vitamine C) diminuent consıdérablement.

Le veau, atteint d'ascaridiase à la naissance est déjà en état de dénutrition, état souvent aggravé par une alimentation insuffisante en quantité. L'amaigrissement est sensible. II suffit d'administrer du Thiabendazole ou du Tétramisole pour voir le poids moyen d'un veau parasité augmenter de 10,5 p. 100 en zone sahélienne (GRABER et Coll., 1968) eł de 13,5 p. 100 d̀ Bouar (BOUCHET, 1969).

Cet amaigrissement s'accompagne d'une anémie souvent intense : le nombre d'hématies diminue de 12 à 17 p. 100 selon les régıons et le bétail en cause. Les eosinophiles sont nombreux et le taux d'hémoglobine basse. D'une façon générale, la croissance du jeune est retardée et, parfois, ce retard peut être d'un an.

La mortalité des veaux de lait imputable au parasitisme gastro-intestinal est difficıle d̀ chiffrer : au Tchad, on l'estime entre 10 et 20 p. 100 de l'effectif. En RCA, selon les troupeaux et les années, elle varie de 1 à 20 p. 100, ces chiffres pouvant d'ailleurs être consıdérés comme valables pour toute la région couverte par l'enquête.

Dans ces conditions, il est bien évident que les helminthiases ef la sous-alimentation chronique interviennent, dès le jeune âge, pour limiter la croissance normale du troupeau. Ce point méritait d'être souligné.

\section{4. - Conclusions.}

Le parasitisme du veau de lait est, dans l'Ovest de la Républıque Centrafricaıne, un problème majeur, à résoudre d'urgence.

L'apparition d'anthelminthiques à large spectre comme le Thiabendazole, le Tétramisole ou le Tartrate de Pyrantel, permettent actuellement de traiter les ascaris, les «Strongles» et, partiellement, les Strongylordes.

L'époque des traitements reste à préciser. D'après les courbes d'infestation et les autopsies (BOUCHET, 1969) le déparasıtage des animaux atteints devrait avoir lieu vers l'âge d'un mois, ce qui permettrait de réduire dans de fortes proportions l'incidence de l'ascaridiase, et de rendre ainsi les veaux plus résistants aux agressions ultérieures par les «Strongles» ou les coccidies.

Les jeunes de 2 à 7 mois se réinfestant très facilement en saison des pivies, il importe de prévoir un second traitement en octobrenovembre pour les animaux nés en fin de saison sèche.

Quant aux coccidies, une enquête plus approfondie s'avère nécessaire de manière à délimiter les zones infestées et la prophylaxie à mettre en ceuvre.

Institut d'Elevoge et de Médecine vétérinare des Pays tropicaux : Laboratoire de Forcha d̀ FortLamy (Tchad) et Centre de Bouar (RCA). Direction de l'Elevage de RCA d̀ Bangui.

\section{SUMMARY}

Zebu parasitism in west Central African Republic. 1. Sucking calves parasitism

In west Central African Republic, the authors report the existence, in 0 to 7 months old sucking calves, of numerous parasites species, associated or not, such as : Neoascaris vifulorum, Strongyloides papillosus, Oesophagostomum (Bosicolo) 
radiatum, Bunostomum phlebotomum, Cooperio punctota and Cooperia pectinato, Haemoncus contortus and different coccidio.

76,4 p. 100 of calves are infested. The infestation rates and the parasitism form are varying with age and season. Mortality fluctuates between 1 and 20 p. 100, according to the herds and the years.

It is an imperative problem to be worked out for the central african animal husbandry.

\section{RESUMEN}

El parasitismo del cebú en el oesle de la República Centroafricana

I. Parasitismo de los ferneros de pecho

En el oeste de la República Centroofricana, los autores señalan la existencia, en los terneros de pecho de 0 a 7 meses de edad, de numerosos parásitos, solos o asociados entre ellos, perteneciendo a las especies siguientes : Neoascoris vitulorum, Strongyloides papillosus, Oesophagostamum (Bosicola) radiatum, Bunostomum phiebotomum, Cooperia punctata y Cooperio pectinato, Haemoncus contortus y varias coccidias.

76,4 p. 100 de los animales estan infestados ; las tasas de infestación y la forma del parasitismo varian según la edad y la estación. La morfalidad oscila entre 1 y 20 p. 100, según los rebaños y los años.

Se trata de un problemo importante, para la ganaderia de la República Centroafricana, necesitando una solución urgente.

\section{BIBLIOGRAPHIE}

1. BOUCHET (A.). - L'enquête épidémıologique en helminthologie vétérinaire. Objet, méthodes. Compte rendu d'observations faites dans un élevage ovin. Thèse vétérinaire, Lyon, $55 \mathrm{p}$.

2. BOUCHET (A.). - Etude, dans certaines conditions africaines, de l'action antiparasitaire du Tétramisole par voie buccale dans I'Ascaridiase du veau de lait. Rap. IEMVT, Centre Bouar, 1969, 28 p.

3. CHABRA (A.). - Aperçu sur le climat centrafricain. Asecna, Bangui, 1962, ronéo, $25 \mathrm{p}$.

4. CVETKOVIC (L.) et NEVENIC (V.). Contribution à la connaissance de l'épizootologie de la Néoascaridiase des veaux. Acto Vet. Beorg., 1960, 10, 4, 49-59.

5. EUZEBY (J.). - Diagnostic expérimental des helminthoses animales. Vigot, Paris, 1958.

6. EUZEBY (J.). - Les maladies vermineuses des animaux domestiques et leurs incidences sur la pathologie humaine. T. I, fasc. 2 Vigot frères, Paris, 1963.

7. GOULEE (A.). - Note sur la pluviométrie en République centrafricaine. Asecna, Bangui 1964, ronéo, $92 \mathrm{p}$.

8. GRABER (M.). - Etude préliminaire sur le parasitisme des animaux domestiques en République centrafricaine. Rapporf IEMVT, Farcha 1961, 82 p.

9. GRABER (M.). TRONCY (P.) et FERNAGUT (R.). - Etude, dans certaines conditions africaines, de l'action antiparasitaire du Thiabendazole sur divers helminthes des animaux domestiques. III. Nématodes du veau de lait. Rev. Elev. Méd. Vét. Pays trop., 1968 (a) 21, 1, 71-8.

10. GRABER (M.). - Etude du « Bebou », parasitose sévissant chez les zébus de la Topia. Moyens de lutte. Rapport IEMVT, Farcha, 1968 (b), $41 \mathrm{p}$.

11. GRABER (M.). - Helminthes ef helminthiases. Bilan d'activités. Rapport Annuel 1967, Laboratoire de Farcha, 1968, 192 p. 